\title{
Piezoelectric Actuators With Integrated High-Voltage Power Electronics
}

\author{
Yuen Kuan Yong, Member, IEEE, and Andrew J. Fleming, Member, IEEE
}

\begin{abstract}
This paper explores the possibility of piezoelectric actuators with integrated high-voltage power electronics. Such devices dramatically simplify the application of piezoelectric actuators since the power electronics are already optimized for the voltage range, capacitance, and power dissipation of the actuator. The foremost consideration is the thermal impedance of the actuator and heat dissipation. Analytical and finite-element methods are described for predicting the thermal impedance of a piezoelectric bender. The predictions are compared experimentally using thermal imaging on a piezoelectric bender with laminated miniature power electronics.
\end{abstract}

Index Terms-Actuators, piezoelectric, power electronics.

\section{INTRODUCTION}

$\mathbf{P}$ IEZOELECTRIC transducers have become ubiquitous in applications requiring precision motion and force control. For example, positioning systems [1]-[4], micromotors [5]-[8], machine tools [9], pumps [10], surgical tools [11], fuel injectors [12], scanning probe microscopes (SPMs) [13]-[16], and vibration control systems [17], [18].

In addition to the high resolution [19], piezoelectric actuators are also desirable for their low mass, dimensions, and zero static power consumption. However, these benefits may be negated by the requirement for an external high-voltage amplifier, power supply, sensors, and a feedback loop. In some applications, feed-forward control can alleviate the need for sensors and a feedback loop [20]-[22], however the driving electronics are still required.

The requirement for an external high-voltage amplifier has significantly limited the feasibility of miniaturized piezoelectric systems. For example, a piezoelectric microgripper still requires bulky support electronics $(100 \mathrm{~mm} \times 100 \mathrm{~mm})$ even though the device itself is only $23 \mathrm{~mm}$ in width [23]. This also necessitates the need for high-voltage wiring between the driver and actuator. Piezoelectric bender actuators have also shown promise in microrobotic applications. In [24], an energy recovery circuit was designed to reduce the size of the drive electronics so that

Manuscript received March 5, 2013; revised July 17, 2013 and December 10, 2013; accepted February 27, 2014. Date of publication March 24, 2014; date of current version October 24, 2014. Recommended by Technical Editor G. Schitter. This work was supported by the Australian Research Council Discovery Project DP0986319 and the DECRA Project DE130100879.

The authors are with the School of Electrical Engineering and Computer Science, The University of Newcastle, Callaghan, N.S.W. 2308, Australia (e-mail: yuenkuan.yong@newcastle.edu.au; andrew.fleming@newcastle.edu.au).

Color versions of one or more of the figures in this paper are available online at http://ieeexplore.iee.org.

Digital Object Identifier 10.1109/TMECH.2014.2311040 they could be mounted on-board the robot. Bimorph actuators have also been used for piezoelectric motors with nanometer resolution [25].

In sensing applications, the conditioning electronics are routinely integrated with the transducer to avoid interference from high-impedance wiring. For example, accelerometers are produced with an integrated FET amplifier [26]. However, it is not trivial to apply such techniques for actuation since the drive electronics are significantly more complicated, require inductive components, and may also dissipate heat.

This study investigates the feasibility of a piezoelectric bimorph actuator with integrated power electronics. Due to the recent availability of high-voltage integrated circuits, it is now feasible to construct a complete high-voltage power supply and amplifier in an area smaller than $7 \mathrm{~mm}^{2}$. The driver can then be thermally attached to the actuator for heat dissipation. This technique is demonstrated in Section II.

The required power dissipation is derived in Section III which defines the required thermal impedance of the actuator from the mounting location to the ambient environment. Analytical and finite-element techniques are applied to estimate the thermal impedance of the actuator in Section IV. The actuator is found to be capable of easily dissipating the required power with negligible erroneous deflection due to thermal expansion. The predictions are compared to experimental results in Section V.

By integrating the power electronics and actuator, the electrical design process is effectively eliminated. This dramatically reduces the cost and complexity of installing piezoelectric actuators in industrial and automotive applications where there may be a restriction on the maximum wiring voltage. This level of miniaturization also creates new possibilities for autonomous piezoelectric walking and flying robots with independent control over each actuator. For example, a six-legged robot could be designed with independent control over each limb from a single 3.7-V battery cell.

\section{EXAMPLE BIMORPH ACTUATOR WITH INTEGRATED DRIVER}

\section{A. Actuator}

The actuator is a two-layer brass reinforced bimorph bender from Piezosystems Inc. (Q220-A4-503YB). Both of the piezoelectric layers are poled in the same direction and driven electrically in parallel, i.e., the top and bottom surfaces are grounded and the internal layer is driven. The bender is fixed onto a mounting base as shown in Fig. 1. The properties of interest are listed in Table I. The static and dynamic behavior of piezoelectric bimorphs is described, for example, in [27]. 


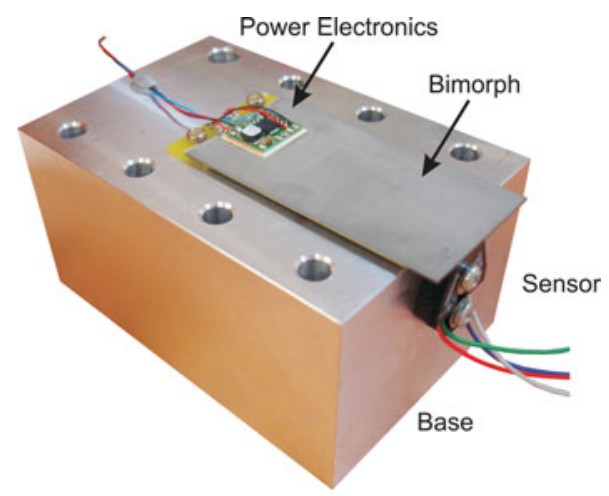

Fig. 1. Piezoelectric bimorph actuator with thermally attached power electronics.

TABLE I

Properties of THE PIEZo Systems Q220-A4-503YB Bender ACtUATOR

\begin{tabular}{cc}
\hline Dimensions & $63.5 \times 31.8 \times 0.51 \mathrm{~mm}$ \\
\hline Piezo Material & $5 \mathrm{~A} 4 \mathrm{E}$ \\
\hline Curie Temperature & $350^{\circ} \mathrm{C}$ \\
\hline Voltage & $\pm 90 \mathrm{~V}$ \\
\hline Mounted Deflection & $\pm 1.26 \mathrm{~mm}$ \\
\hline Mounted Stiffness & $245 \mathrm{~N} / \mathrm{m}$ \\
\hline Mounted Blocking Force & $0.31 \mathrm{~N}$ \\
\hline Mounted Resonance Freq. & $68 \mathrm{~Hz}$ \\
\hline Capacitance & $260 \mathrm{nF}$ \\
\hline
\end{tabular}

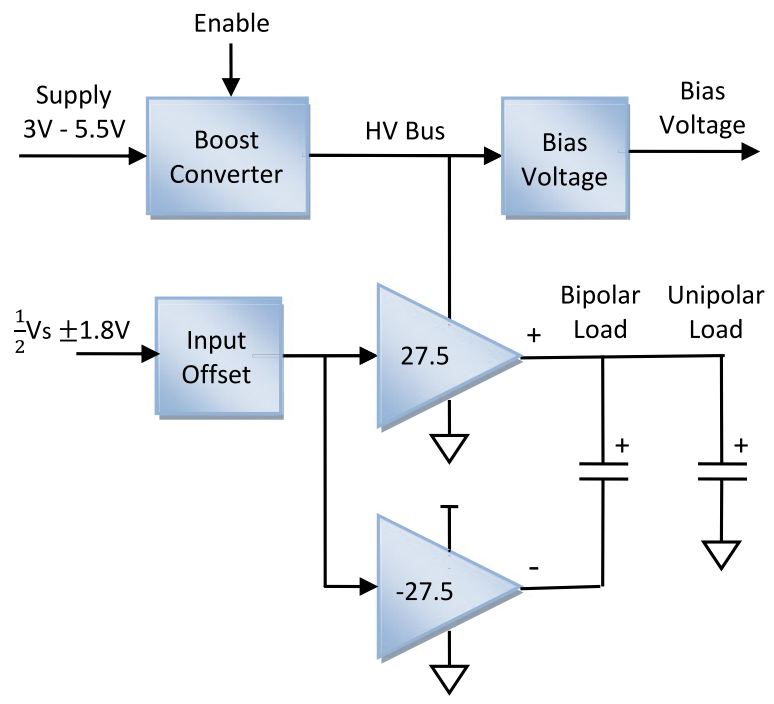

Fig. 2. Power electronics functionality [28].

\section{B. Power Electronics}

The power electronic module is a PiezoDrive PDu100 driver [28] illustrated in Fig. 2. The power supply is a closed-loop boost converter that produces $105 \mathrm{~V}$ from a 3 to $5.5 \mathrm{~V}$ supply. The supply voltage is compatible with a single Lithium Ion or Polymer battery cell. To obtain a bipolar output voltage, the load is driven by two linear amplifiers with opposite gain, effectively doubling the output voltage range to $\pm 100 \mathrm{~V}$.

The circuit and support electronics are mounted on a thermally conductive PCB with an area of $12 \mathrm{~mm}^{2}$. Approximately

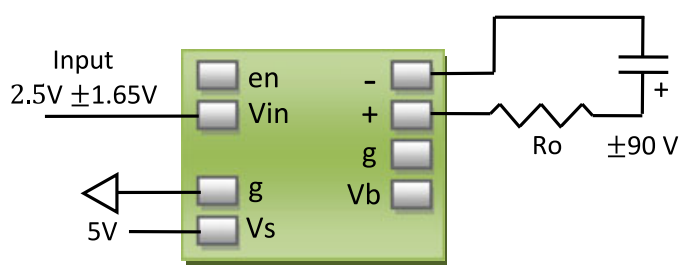

Fig. 3. Connection diagram of the power electronics and actuator.

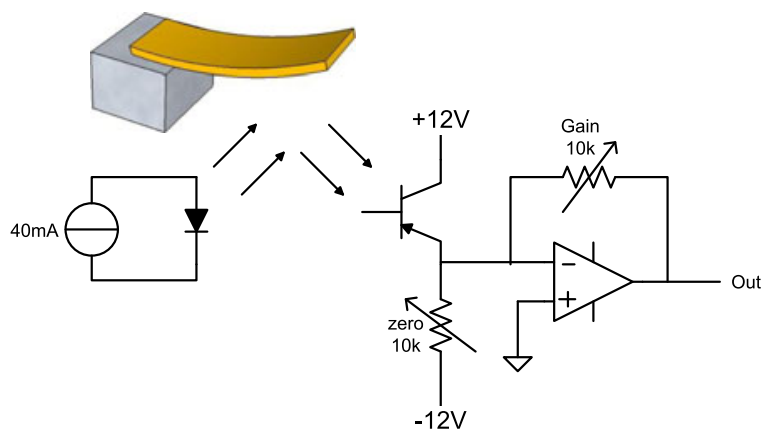

Fig. 4. Displacement sensor schematic diagram.

half the area is used for connecting pads so the size could be reduced to less than $7 \mathrm{~mm}^{2}$ if necessary. These dimensions are compatible with many of bender geometries.

The module is bonded to the actuator using an adhesive thermally conductive insulator (T-Global LI-98-150-025). This material is a soft elastomer which effectively decouples the strain of the actuator from the high-stiffness of the PCB. Three layers of $0.25 \mathrm{~mm}$ thickness were used for a total thickness of $0.75 \mathrm{~mm}$.

The connection diagram for the electronics and actuator is shown in Fig. 3. An input signal of $\pm 1.65 \mathrm{~V}$ with an offset of $2.5 \mathrm{~V}$ produces a $\pm 90-\mathrm{V}$ output signal which is the full range of the actuator.

\section{Sensor Design}

The position is measured with an OPB703 reflective sensor and the conditioning circuit illustrated in Fig. 4. When driven with a $40 \mathrm{~mA}$ current, the phototransistor current is proportional to the distance over a range of approximately $2.5 \mathrm{~mm}$ (centered at $2.5 \mathrm{~mm}$ ). The op-amp acts as a photocurrent amplifier with a variable gain up to $10 \mathrm{~V} / \mathrm{mA}$. The zero potentiometer is adjusted so that the output is zero volts with zero deflection. Other possible sensor configurations are discussed in [29].

\section{ELECTRICAL CONSIDERATIONS}

For the purposes of estimating electronic power dissipation at frequencies below the first resonance, a piezoelectric actuator can be adequately modeled as a capacitor as shown in Fig. 5(a) [30]. It should be noted that soft piezoceramics, such as PZT-5H, exhibit significant hysteresis that can effectively double the small-signal capacitance when driven at full range.

The power dissipation of the grounded amplifier in Fig. 5(a) can be found by summing the power supplied by the positive 


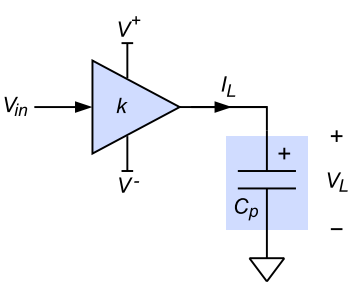

(a)

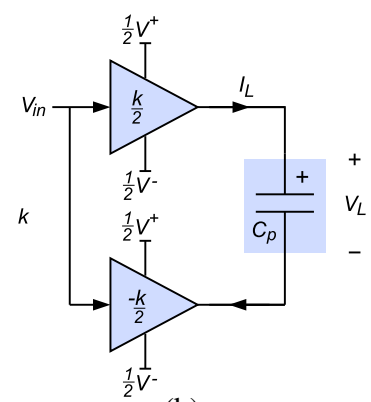

(b)
Fig. 5. (a) Electrical model of a piezoelectric actuator driven in the grounded configuration and (b) the equivalent bridged configuration.

and negative supplies [31],

$$
P_{d}=V^{+} I_{a v}^{+}+V^{-} I_{a v}^{-} .
$$

For a capacitive load $I_{a v}^{+}=-I_{a v}^{-}$; therefore,

$$
P_{d}=\left(V^{+}-V^{-}\right) I_{a v} .
$$

With a sinusoidal excitation, the peak load current is

$$
I_{L(p k)}=\pi V_{L(p-p)} f C_{p} .
$$

The average current is, therefore,

$$
I_{a v}=\frac{1}{2 \pi} \int_{0}^{\pi} I_{L(p k)} \sin (\theta) d \theta=\frac{I_{L(p k)}}{\pi} .
$$

Thus, the power dissipation is

$$
P_{d}=\left(V^{+}-V^{-}\right) V_{L(p-p)} C_{p} f .
$$

The driver described in Section II-B operates in the bridged configuration as shown in Fig. 5(b). To achieve the same load voltage as the grounded circuit, the bridged configuration requires only half the supply voltage. However, since the average load current is identical and there are two amplifiers, the total power dissipation of the bridged configuration is identical to the grounded circuit.

In addition to the power dissipated by the amplifiers, the finite efficiency of the boost converter must also be considered. If the converter efficiency is $\eta$, the total power dissipation becomes

$$
P_{d}=\frac{1}{\eta}\left(V^{+}-V^{-}\right) V_{L(p-p)} C_{p} f .
$$

The previous equation is relevant to any linear amplifier with a switched mode power supply operating in the standard or bridged configuration. It should be noted that $\eta$ is the fullload converter efficiency. The actual efficiency may vary under lighter load conditions; however, this approximation is sufficient for estimating the worst case power dissipation. For the bridged driver described in Section II-B, $V^{+} / 2=105 \mathrm{~V}, V^{-}=0 \mathrm{~V}$, and the efficiency is approximately $\eta=0.75$. Therefore, the power dissipation is

$$
P_{d}=\frac{210 \mathrm{~V} \times 520 n F}{0.75} V_{L(p-p)} f .
$$

With the full-scale $\pm 90 \mathrm{~V}$ load voltage, the power dissipation is $26 \mathrm{~mW} / \mathrm{Hz}$. Thus, if the actuator is operated at the maximum

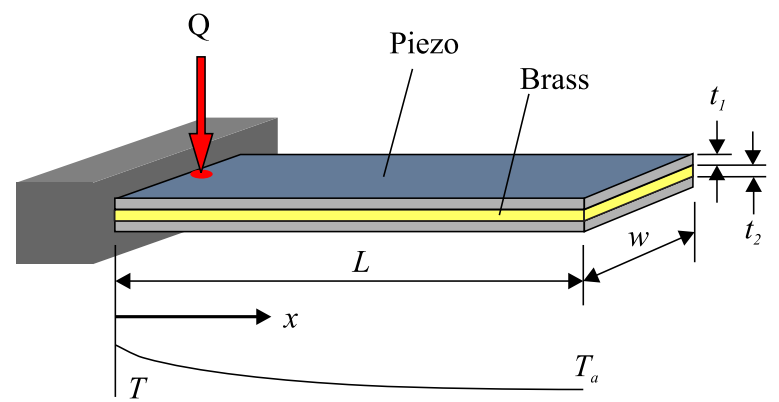

Fig. 6. Cantilever beam model with the fixed-end heated.

operating frequency of $60 \mathrm{~Hz}$, the maximum power dissipation is approximately $1.5 \mathrm{~W}$.

\section{THERMAL DISSIPATION}

As discussed previously, the power electronic module may dissipate up to $1.5 \mathrm{~W}$. The thermal impedance of the mounting location must be low enough to ensure that the junction temperature of the electronics does not exceed $150{ }^{\circ} \mathrm{C}$. Although the Curie temperature of the piezoelectric material is $350{ }^{\circ} \mathrm{C}$, the maximum practical temperature is also limited to around $150^{\circ} \mathrm{C}$ by the glue layers. To investigate the thermal dissipation of the bender, the thermal impedance is calculated analytically in this section. This result is compared to an ANSYS finite-element (FE) simulation and experimental results. The thermally induced deflection and extension of the bender is also studied by finite-element analysis.

\section{A. Analytical Estimation}

To estimate the thermal impedance, the temperature distribution of the bender is first calculated. The bimorph bender is thermally modeled as a fin. The Biot number $\left(\mathrm{Bi}=h_{c} t / 2 k_{a}\right)$ of the bender is estimated to be $<0.1$, where $h_{c}$ is the natural convection coefficient, $t$ is the thickness, and $k_{a}$ is the average thermal conductivity of the bender. $\mathrm{Bi}<0.1$ shows that the bender has a uniform temperature along its thickness direction where temperature at the center will not differ from that of the surface by more than $5 \%$ [32]. Therefore, the temperature distribution along the thickness direction can be ignored, and only the temperature distribution along the longitudinal dimension is considered. As a result, the bender is modeled as 1-D fin.

As shown in Fig. 6, the bimorph is a cantilever beam with the fixed-end heated by the integrated power electronics. The maximum power is assumed to be $Q=1.65 \mathrm{~W}$. $T_{a}$ is the ambient temperature, $L$ is the length, $w$ is the width, and $x$ is the distance along the length of the bender. The temperature distribution profile of the bender along the $x$-direction can be found by solving the following differential equation [32], [33],

$$
\frac{d^{2} T}{d x}-\beta^{2}\left[T(x)-T_{a}\right]=0
$$

where $\beta^{2}=2 h_{c} /\left(2 k_{1} t_{1}+k_{2} t_{2}\right), k_{1}$ and $t_{1}$ are the thermal conductivity and thickness of the piezoelectric layer respectively, $k_{2}$ and $t_{2}$ are the thermal conductivity and thickness of 
TABLE II

DiMENSIONS AND PROPERTIES OF THE BENDER

\begin{tabular}{cccccc}
\hline $\begin{array}{c}L \\
\mathrm{~mm}\end{array}$ & $\begin{array}{c}w \\
\mathrm{~mm}\end{array}$ & $\begin{array}{c}t_{1} \\
\mathrm{~mm}\end{array}$ & $\begin{array}{c}t_{2} \\
\mathrm{~mm}\end{array}$ & $\begin{array}{c}k_{1} \\
\mathrm{~W} / \mathrm{mK}\end{array}$ & $\begin{array}{c}k_{2} \\
\mathrm{~W} / \mathrm{mK}\end{array}$ \\
\hline 63.5 & 31.8 & 0.2 & 0.1016 & 1.25 & 115 \\
\hline
\end{tabular}

the brass layer, respectively. Since $\beta$ is a constant, (8) has the solution

$$
T(x)-T_{a}=C_{1} e^{-\beta x}+C_{2} e^{\beta x} .
$$

Assuming the thermal heating occurs at $x=0$, we have a boundary condition

$$
\begin{aligned}
-\left(2 k_{1} A_{1}+k_{2} A_{2}\right) \frac{d T(0)}{d x} & =Q \\
-w\left(2 k_{1} t_{1}+k_{2} t_{2}\right) \frac{d T(0)}{d x} & =Q \\
\frac{d T(0)}{d x} & =\frac{-Q}{w\left(2 k_{1} t_{1}+k_{2} t_{2}\right)}
\end{aligned}
$$

where $A_{1}$ and $A_{2}$ are the cross-sectional area of the piezoelectric and brass layer, respectively. Differentiating (9), and equate it to $(10)$, we have

$$
C_{2}=C_{1}-\frac{Q}{\beta w\left(2 k_{1} t_{1}+k_{2} t_{2}\right)} .
$$

Substituting (11) into (9), and by assuming that at $x=$ $L, T(L)=T_{a}$, we have

$$
C_{1}=\frac{Q e^{2 \beta L}}{\beta w\left(2 k_{1} t_{1}+k_{2} t_{2}\right)\left(1+e^{2 \beta L}\right)} .
$$

Substituting (12) into (11), we have

$$
C_{2}=\frac{-Q}{\beta w\left(2 k_{2} t_{1}+k_{2} t_{2}\right)\left(1+e^{2 \beta L}\right)} .
$$

Equations (12) and (13) can be simplified to

$$
\begin{aligned}
& C_{1}=\frac{Q e^{\beta L}}{2 \beta w\left(2 k_{1} t_{1}+k_{2} t_{2}\right) \cosh (\beta L)} \\
& C_{2}=\frac{-Q e^{-\beta L}}{2 \beta w\left(2 k_{1} t_{1}+k_{2} t_{2}\right) \cosh (\beta L)} .
\end{aligned}
$$

Substituting (14) and (15) into (9), the temperature distribution along the length direction of the bender is expressed as

$$
T(x)-T_{a}=\frac{\sinh [\beta(L-x)]}{\cosh (\beta L)} \frac{Q}{\beta w\left(2 k_{1} t_{1}+k_{2} t_{2}\right)} .
$$

The dimensions and thermal properties of the bender are listed in Table II. The heat flow applied to the fixed-end is $Q=1.65 \mathrm{~W}$ and the ambient temperature is $T_{a}=295 \mathrm{~K}$. With a convection coefficient of $h_{c}=28 \mathrm{~W} / \mathrm{m}^{2} \mathrm{~K}$, the predicted temperature at the fixed-end is $84.8^{\circ} \mathrm{C}$. The temperature distribution along $x$ is plotted in Fig. 7. At $x=L$, both the FE simulated and analytical temperature results approach $T_{a}$, which justify the assumption made in (12) and (13). The analytical temperature profile differs slightly from the FE result due to the one-dimensional (1-D) analytical model and the chosen boundary condition where input power was applied at $x=0$. The FE model is a 3-D model

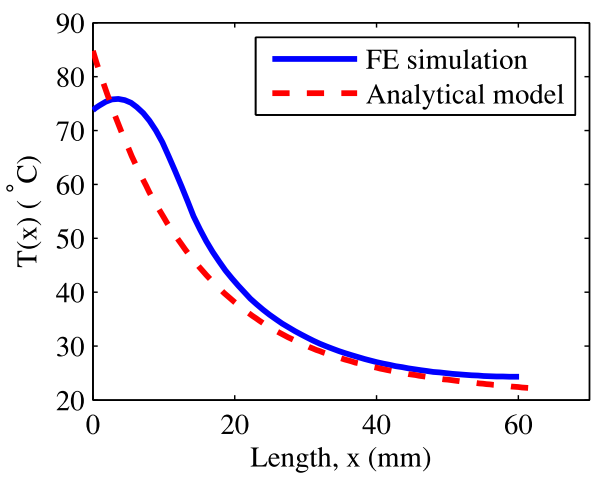

Fig. 7. Temperature distribution profile $T(x)$ of the bender.

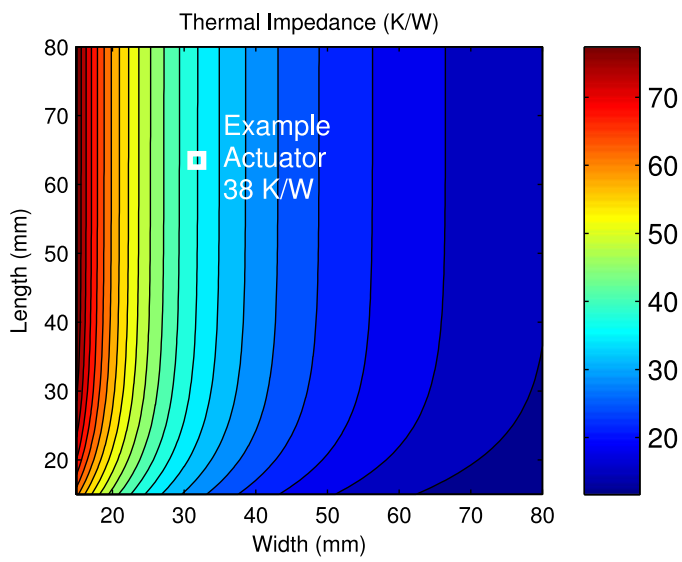

Fig. 8. Base thermal impedance versus the length and width of a rectangular bender. The dimensions of the actuator under consideration are marked with a white box.

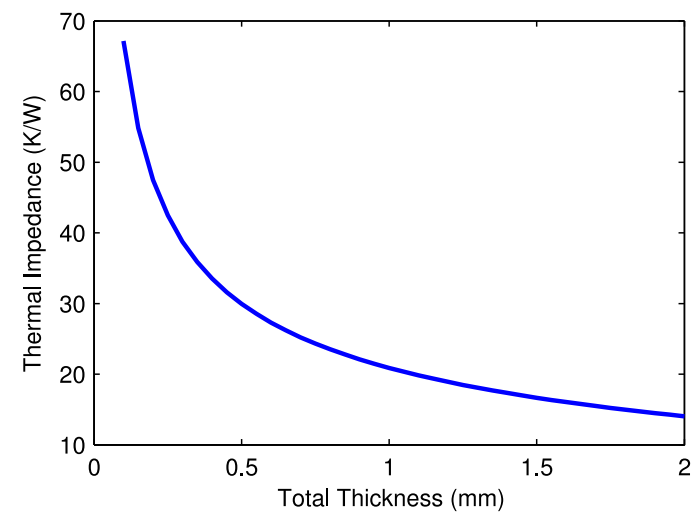

Fig. 9. Base thermal impedance versus the total thickness of a rectangular bimorph actuator (with equal thickness of piezoelectric and metal layers).

where input power was applied on a $12 \mathrm{~mm}^{2}$ area. It is possible to extend the analytical model to multidimensional. However, given the approximate nature of the model, the extension is not deemed justified.

Based on the temperature difference, the thermal impedance of the bender from the applied heat source to ambient is estimated by $Z=\left[T(0)-T_{a}\right] / Q=38 \mathrm{~K} / \mathrm{W}$. This result shows that the piezoelectric bender can be used to dissipate two to three Watts of heat, depending on the ambient temperature. 


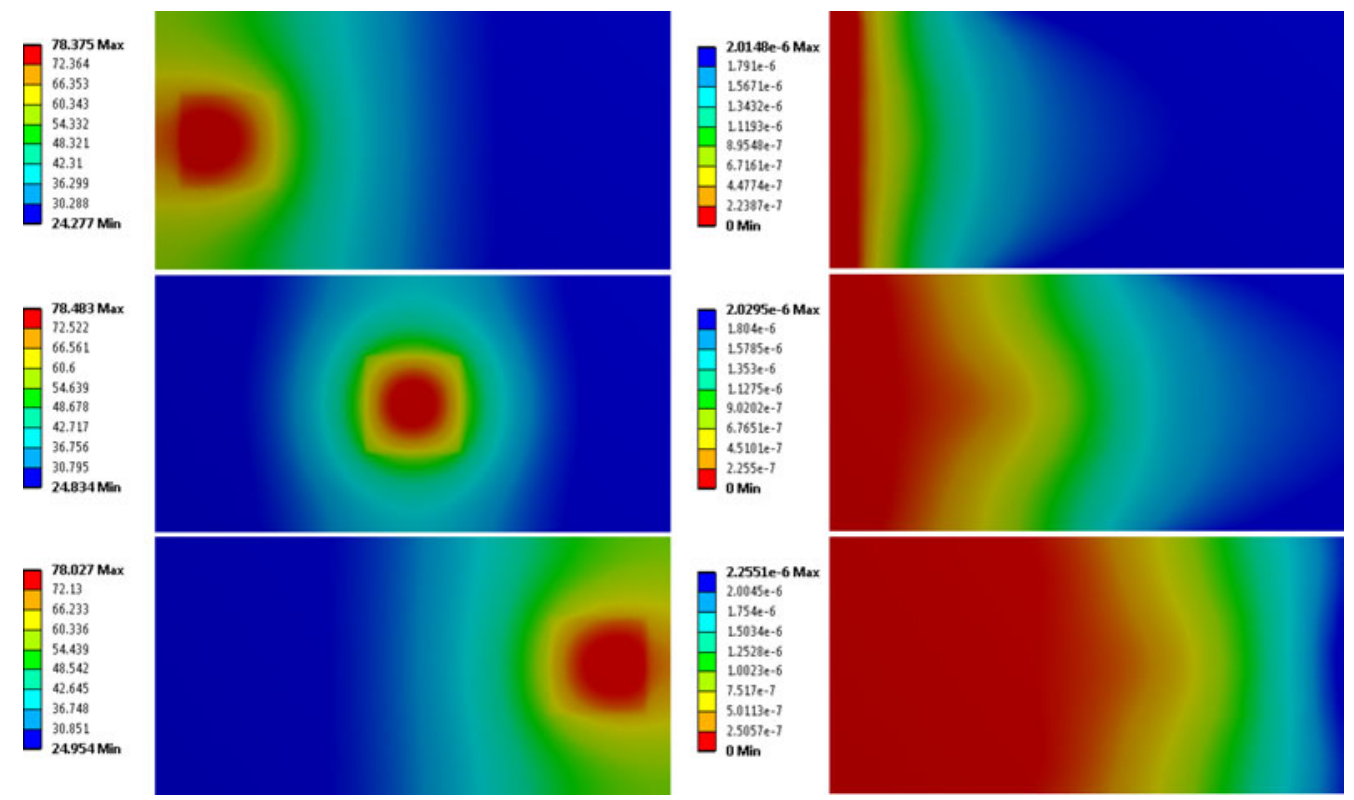

Fig. 10. FE-simulated temperature profiles in degree Celsius (left column) and the corresponding deflections in meter (right column) of the bender. Note that the deflection results are along the bending direction of the bender. Heat source was applied at the (top) fixed-end, (center) middle, and (bottom) free-end of the bender.

When there is a high power dissipation, the actuator will also be moving quickly which is advantageous since this promotes air flow and better cooling. This is particularly true of actuators with large lateral motions such as benders.

To examine the effect of dimensions, the thermal impedance is plotted against the length and width in Fig. 8. The actuator width has the greatest effect on the thermal impedance since twice the heat can be conducted along the width compared to the length. From Fig. 8, it can be observed that there is not a significant advantages in making the width greater than $50 \mathrm{~mm}$ or the length greater than $25 \mathrm{~mm}$.

The effect of thickness is also examined in Fig. 9. The thickness is observed to have a significant effect on the thermal impedance, primarily due to the increased cross-sectional area of the metal layer. The practical range of thickness is approximately 0.4 to $1 \mathrm{~mm}$.

\section{B. Finite-Element-Analysis of the Piezoelectric Bender}

An FE model of the bender was constructed in ANSYS Workbench to simulate the temperature distribution and deflection of the bender. A heat source was applied at three different locations: the fixed-end, the center, and the free-end of the bender.

Steady-state thermal analysis was first conducted on the piezoelectric bender to simulate the temperature distribution. The piezoelectric and brass layers were modeled using SOLID90 elements. This element has 20 nodes with a single degree of freedom (DOF), that is temperature, at each node. The thermal properties of the piezoelectric and brass layers are listed in Table II. The resulting temperature profiles are plotted in Fig. 10.

After finding the temperature profile, the results were imported into the static structural analysis. In this analysis system, the piezoelectric and brass layers were modeled using
SOLID186 elements. This element has 20 nodes with three DOFs per node: translation in the nodal $x$-, $y$-, and $z$-directions. The FE-simulated temperature profiles and deflections due to temperature variations are plotted in Fig. 10.

Fig. 10 shows that the maximum and minimum temperatures of the bender are very similar for all three heat source locations. The estimated thermal impedances are $32.8 \mathrm{~K} / \mathrm{W}, 32.5 \mathrm{~K} / \mathrm{W}$, and $32.2 \mathrm{~K} / \mathrm{W}$ respectively for the heated fixed-end, middle, and free-end of the bender. These estimated thermal impedances are in close agreement with that of the analytical value.

The simulated maximum deflection is approximately $2 \mu \mathrm{m}$ along the length of the bender for all three cases. Deflections along the bending direction are two orders of magnitude smaller than that of the longitudinal direction. It can be concluded that the unwanted deflections due to heating can be neglected. Since the thermal dissipation and deflection of the bender are not affected by the locations of the heat source, the power electronics can be located at the fixed-end of the bender which is a convenient choice.

\section{EXPERIMENTAL RESULTS}

To simulate the maximum feasible power dissipation, the actuator was driven at $100 \mathrm{~Hz}$ with a $\pm 40 \mathrm{~V}$ sinusoid. This resulted in a supply current of $0.33 \mathrm{~A}$ from a $5 \mathrm{~V}$ supply which indicates a power dissipation of $1.65 \mathrm{~W}$. The resulting temperature profiles were recorded with an FLIR i3 infrared camera.

The temperature profiles for the fixed-end and centrally mounted electronics are shown in Fig. 11. The maximum temperatures were $82{ }^{\circ} \mathrm{C}$ and $84{ }^{\circ} \mathrm{C}$, respectively (with a room temperature of $24{ }^{\circ} \mathrm{C}$ ). Therefore, the thermal impedance was $35 \mathrm{~K} / \mathrm{W}$ for the fixed-end and $36 \mathrm{~K} / \mathrm{W}$ for the central location. These figures correlate well with the predicted thermal impedances of $32.8 \mathrm{~K} / \mathrm{W}$ and $32.5 \mathrm{~K} / \mathrm{W}$. The slightly higher 


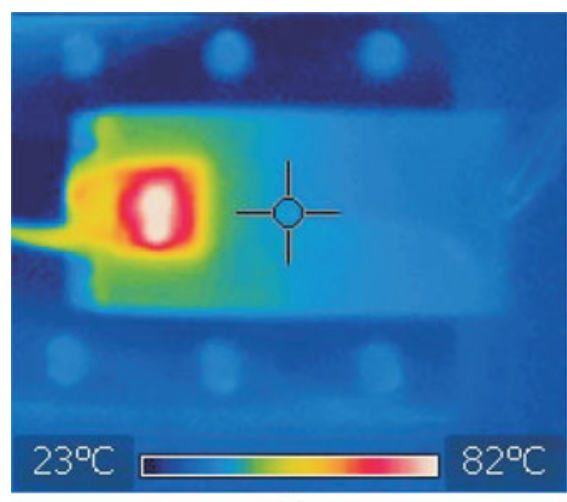

(a)

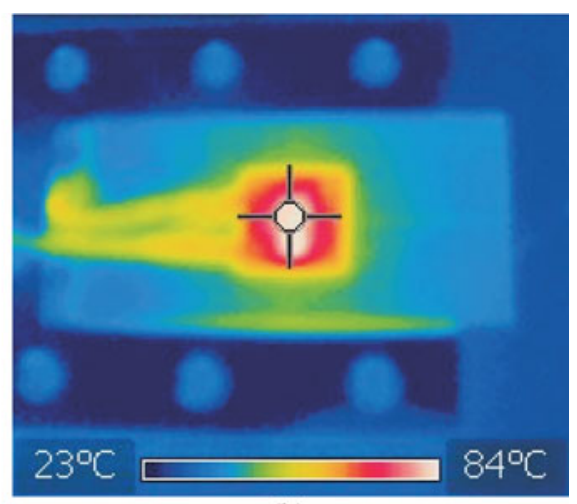

(b)

Fig. 11. Experimental temperature distribution with the electronics located at (a) the fixed end and (b) center.

experimental impedance is due to the impedance of the bonding layer which is predicted to be approximately $6 \mathrm{~K} / \mathrm{W}$, this was not modeled in the FE analysis.

The position sensor described in Section II-C was calibrated to provide a sensitivity of $1.385 \mathrm{~mm} / \mathrm{V}$. The full displacement range of the actuator (without power electronics) was measured to be $2.4 \mathrm{~mm}$ (p-p) with a $5 \mathrm{~Hz} \pm 90 \mathrm{~V}$ sine wave input. When the electronics were mounted in the middle of the actuator, the range was reduced by $5.8 \%$ to $2.26 \mathrm{~mm}$. Similarly, when the electronics were located at the base, the range was reduced by $4.2 \%$ to $2.3 \mathrm{~mm}$. These range reductions are not considered to be significant since they are smaller than the range tolerance specified by the manufacturer. However, the range reduction could be reduced by using a thermal interface material with greater elasticity.

To assess the thermally induced bending, the actuator frequency was increased until the input electrical power was $1.65 \mathrm{~W}$. After the temperature reached a maximum, the frequency was reduced to $5-\mathrm{Hz}$ while monitoring the average value of the position signal. A $24-\mathrm{mV}$ change in the dc offset was observed which corresponds to a thermally induced deflection of $0.033 \mathrm{~mm}$ or $1.4 \%$ of the range. This is larger than the FEA prediction of $0.1 \%$. Since there is little temperature difference between the top and bottom surface of the actuator, the additional deflection is thought to be caused by the thermal strain of the thermal interface material and PCB, which was not modeled in the FEA. The thermally induced strain of the electronics could be eliminated by mounting a second amplifier on the bottom of the actuator. With the two amplifiers connected in series, the dissipated power would be equal and the thermally induced strains would be equal and opposite. If the $1.4 \%$ deflection is deemed to be significant, the electronics could also be mounted closer to the free end.

\section{CONCLUSION}

This paper has described a piezoelectric bender with integrated high-voltage power electronics. Although the power electronics require heat dissipation, the high thermal conductivity of the piezoelectric ceramic is found to provide effective heat sinking.
The power electronics for an example bimorph actuator required $26 \mathrm{~mW} / \mathrm{Hz}$ of power dissipation. Since the thermal impedance of the actuator was found to be $32 \mathrm{~K} / \mathrm{W}$, the temperature of the electronics and actuator will remain in the safe region, even when driving the actuator at full-range near the resonance frequency. The bending error caused by thermal expansion is negligible due to the almost uniform temperature distribution through the thickness of the bender.

With integrated electronics, only three wires are required for the actuator: $+5 \mathrm{~V}$, ground, and the input signal. This dramatically simplifies the application of piezoelectric actuators and eliminates the need for high-voltage wiring and power supplies. Applications include autonomous piezoelectric robotics, industrial actuators, micropumps, positioning systems, and vibration control.

Future work includes replacing the linear amplifiers with an $\mathrm{H}$-Bridge to improve efficiency. The final goal is to produce a driver smaller than $10 \mathrm{~mm}^{2}$ with power-electronics, a sensor interface, and digital feedback loop. This approach will produce a true mechatronic system with all of the required control and drive electronics. Another goal is to assess the impact of thermal cycling on the fatigue life and performance of the actuators.

\section{REFERENCES}

[1] Y. K. Yong, S. O. R. Moheimani, B. J. Kenton, and K. K. Leang, "Invited review article: High-speed flexure-guided nanopositioning: Mechanical design and control issues," Rev. Sci. Instrum., vol. 83, no. 12, p. 121101, 2012.

[2] A. A. Eielsen, J. T. Gravdahl, and K. Y. Pettersen, "Adaptive feed-forward hysteresis compensation for piezoelectric actuators," Rev. Sci. Instrum., vol. 83, no. 8, p. 085001, 2012.

[3] E. Guliyev, T. Michels, B. Volland, T. Ivanov, M. Hofer, and I. Rangelow, "High speed quasi-monolithic silicon/piezostack SPM scanning stage," Microelectron. Eng., vol. 98, pp. 520-523, 2012.

[4] A. J. Fleming, "Nanopositioning system with force feedback for highperformance tracking and vibration control," IEEE/ASME Trans. Mechatronics, vol. 15, no. 3, pp. 433-447, Jun. 2010.

[5] K. Uchino and J. R. Giniewica, Micromechatronics. New York, NY, USA: Marcel Dekker, 2003.

[6] Y. Zhang, G. Liu, and J. Hesselbach, "On development of a rotary linear actuator using piezoelectric translators," IEEE/ASME Trans. Mechatronics, vol. 11, no. 5, pp. 647-650, Oct. 2006.

[7] P. Tenzer and R. Mrad, "A systematic procedure for the design of piezoelectric inchworm precision positioners," IEEE/ASME Trans. Mechatronics, vol. 9, no. 2, pp. 427-435, Jun. 2004. 
[8] R. Merry, N. de Kleijn, M. van de Molengraft, and M. Steinbuch, "Using a walking piezo actuator to drive and control a high-precision stage," IEEE/ASME Trans. Mechatronics, vol. 14, no. 1, pp. 21-31, Feb. 2009.

[9] Y. Takeuchi, Y. Sakaida, K. Sawada, and T. Sata, "Development of a 5axis control ultraprecision milling machine for micromachining based on non-friction servomechanisms," CIRP Ann.-Manufactur. Technol., vol. 49, no. 1, pp. 295-298, 2000.

[10] L. D. Mauck and C. S. Lynch, "Piezoelectric hydraulic pump development," J. Intell. Mater. Syst. Struct., vol. 11, no. 10, pp. 758-764, 2000.

[11] M. Robiony, F. Polini, F. Costa, N. Zerman, and M. Politi, "Ultrasonic bone cutting for surgically assisted rapid maxillary expansion (SARME) under local anaesthesia," Int. J. Oral Maxillofac. Surg., vol. 36, no. 3, pp. 267-269, 2007.

[12] D. Mehlfeldt, H. Weckenmann, and G. Sthr, "Modeling of piezoelectrically actuated fuel injectors," Mechatronics, vol. 18, no. 5-6, pp. 264-272, 2008.

[13] S. M. Salapaka and M. V. Salapaka, "Scanning probe microscopy," IEEE Control Syst. Mag., vol. 28, no. 2, pp. 65-83, Apr. 2008.

[14] G. Schitter, P. J. Thurner, and P. K. Hansma, "Design and input-shaping control of a novel scanner for high-speed atomic force microscopy," Mechatronics, vol. 18, no. 5-6, pp. 282-288, Jun. 2008.

[15] Y. K. Yong, A. J. Fleming, and S. O. R. Moheimani, "A novel piezoelectric strain sensor for simultaneous damping and tracking control of a highspeed nanopositioner," IEEE/ASME Trans. Mechatronics, vol. 18, no. 3, pp. 1113-1121, Jun. 2012.

[16] B. J. Kenton, A. J. Fleming, and K. K. Leang, "A compact ultra-fast vertical nanopositioner for improving SPM scan speed," Rev. Sci. Instrum., vol. 82, no. 12, pp. 123 703-1-123 703-8, 2011.

[17] A. Preumont, Mechatronics, Dynamics of Electromechanical and Piezoelectric Systems. Dordrecht, The Netherlands: Springer-Verlag, 2006.

[18] S. S. Aphale, A. J. Fleming, and S. O. R. Moheimani, "Integral control of resonant systems with collocated sensor-actuator pairs," Smart Mater. Struct., vol. 16, pp. 439-446, Apr. 2007.

[19] A. J. Fleming, "A method for measuring the resolution of nanopositioning systems," Rev. Sci. Instrum., vol. 83, no. 8, p. 086101, 2012.

[20] G. M. Clayton, S. Tien, K. K. Leang, Q. Zou, and S. Devasia, "A review of feedforward control approaches in nanopositioning for high-speed SPM," J. Dyn. Syst., Meas., Control, vol. 131, pp. 061 101-1-061 101-19, Nov. 2009.

[21] K. K. Leang, Q. Zou, and S. Devasia, "Feedforward control of piezoactuators in atomic force microscope systems," IEEE Control Syst. Mag., vol. 29, no. 1, pp. 70-82, Feb. 2009.

[22] H. Jiang, H. Ji, J. Qiu, and Y. Chen, "A modified Prandtl-Ishlinskii model for modeling asymmetric hysteresis of piezoelectric actuators," IEEE Trans. Ultrason., Ferroelectr. Freq. Control, vol. 57, no. 5, pp. 12001210, May 2010

[23] D. Wang, Q. Yang, and H. Dong, "A monolithic compliant piezoelectricdriven microgripper: Design, modeling, and testing," IEEE/ASME Trans. Mechatronics, vol. 18, no. 1, pp. 138-147, Feb. 2013.

[24] B. Edamana and K. Oldham, "Optimal low-power piezoelectric actuator control with charge recovery for a microrobotic leg," IEEE/ASME Trans. Mechatronics, vol. 18, no. 1, pp. 251-262, Feb. 2013.

[25] R. Merry, M. Maassen, M. van de Molengraft, N. van de Wouw, and M. Steinbuch, "Modeling and waveform optimization of a nano-motion piezo stage," IEEE/ASME Trans. Mechatronics, vol. 16, no. 4, pp. 615626, Aug. 2011.

[26] F. Levinzon, "Ultra-low-noise seismic piezoelectric accelerometer with integral fet amplifier," IEEE Sensors J., vol. 12, no. 6, pp. 2262-2268, Jun. 2012.

[27] R. G. Ballas, Piezoelectric Multilayer Beam Bending Actuators. Berlin, Germany: Springer-Verlag, 2007.

[28] PiezoDrive. (2012). "PDu100 datasheet," [Online]. Available: www. piezodrive.com
[29] A. J. Fleming, "A review of nanometer resolution position sensors: Operation and performance," Sens. Actuators A, Phys., vol. 190, pp. 106-126, Feb. 2013.

[30] H. J. M. T. A. Adriaens, W. L. de Koning, and R. Banning, "Modeling piezoelectric actuators," IEEE/ASME Trans. Mechatronics, vol. 5, no. 4 pp. 331-341, Dec. 2000.

[31] A. J. Fleming, "A MHz bandwidth dual-amplifier for driving piezoelectric actuators and other highly capacitive loads," Rev. Sci. Instrum., vol. 80, pp. 104 701-1-104 701-7, 2009.

[32] A. F. Mills, Heat Transfer, 2nd ed. Englewood Cliffs, NJ, USA: PrenticeHall, 1999.

[33] M. Toda, T. Ono, F. Liu, and I. Voiculescu, "Evaluation of bimaterial cantilever beam for heat sensing at atmospheric pressure," Rev. Sci. Instrum., vol. 81 , no. 5 , p. $055104,2010$.

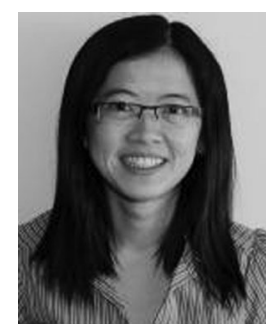

Yuen Kuan Yong (M’09) received the B.Eng. degree (1st Class Hons.) in mechatronic engineering and the $\mathrm{Ph} . \mathrm{D}$. degree in mechanical engineering from The University of Adelaide, Adelaide, Australia, in 2001 and 2007, respectively.

She is currently an Australian Research Council DECRA Fellow with the School of Electrical Engineering and Computer Science, The University of Newcastle, Callaghan, Australia. Her research interests include the design and control of nanopositioning systems, high-speed atomic force microscopy, finiteelement analysis of smart materials and structures, and sensing and actuation.

Dr. Yong received the 2008 IEEE/ASME International Conference on Advanced Intelligent Mechatronics (AIM) Best Conference Paper Finalist Award. She is a member of the Technical Program Committee of AIM and the International Conference on Manipulation, Manufacturing, and Measurement on the Nanoscale (3M-NANO). She is also an Associate Editor of the Editorial Board of the International Journal of Advanced Robotic Systems.

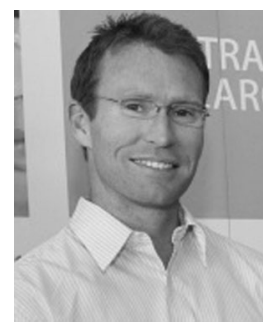

Andrew J. Fleming (M'02) received the Bachelor of Electrical Engineering and Ph.D. degrees from The University of Newcastle, Callaghan, Australia, in 2000 and 2004, respectively.

$\mathrm{He}$ is currently an Australian Research Council Future Fellow and the Director of the Precision Mechatronics Laboratory, The University of Newcastle. His research interests include nanofabrication, microrobotics, metrological sensing, nano-positioning, and high-speed scanning probe microscopy. He is the coauthor of three books and more than 100 Journal and Conference papers. He is the inventor of several patent applications.

Dr. Fleming received the IEEE TRANSACTIONS ON CONTROL SYSTEMS TECHNOLOGY Outstanding Paper Award, The Australian Control Conference Best Student Paper Award, The University of Newcastle Researcher of the Year Award, and the Faculty of Engineering and Built Environment Award for Research Excellence. In 2012, he received the Newcastle Innovation Rising Star Award for Excellence in Research Commercialization. 